\title{
The mixed telemetry/image USN in the overload conditions
}

\author{
Ammar Muthanna*, Andrey Prokopiev**, Andrey Koucheryavy**** \\ * State University of Telecommunication, Pr. Bolshevikov, 22, St.Petersburg, Russia \\ **UbiTel, Ruzovskaya, 21, St.Petersburg, Russia \\ ***Central Science Research Telecommunication Institute, First Proezd Perova Polya, 8, Moscow. Russia \\ ammarexpress@gmail.com, a.prokopiev@ubitel.ru, akouch@mail.ru
}

\begin{abstract}
The Internet of Things (IoT) is a new concept for telecommunication development. The IoT and things determinations are considering in accordance the ITU-T recommendations. The Ubiquitous Sensor Network (USN) is one of the general IoT components. The traffic models for such network are the important field of research presently. The mixed telemetry/image USN behavior in the overload conditions is the investigation goal of this paper. This investigated USN is multiapplication network with AODV (Ad Hoc On Demand Distance Vector) signaling protocol. The paper results show that the USN efficiency can reduce in the overload conditions likely the same effect for the PSTN and NGN networks.
\end{abstract}

Keywords- IoT, USN traffic, image applications, Hurst parameter

\section{INTRODUCTION}

The Internet of Things (IoT) is the new ITU-T concept for the network development. The IoT will be based on the Ubiquitous Sensor Network (USN) [1, 2]. So the USN traffic models should be studied well. The USN traffic models considered for telemetry applications in [3], for medical applications in [4]. The image applications are very special for USN in according with the necessary big data capacity for trans-mission and rates. Usually, the USN for the image applications includes the telemetry sensor nodes too. The USN traffic models for mixed telemetry/image application were studied in [5].

There are many investigations which proposed difference image compressions methods for efficient image transmission in the USN [6, 7, 8]. All of these methods used for special USN, named visual sensor networks or camera sensor networks [9, 10]. The traffic investigation for camera sensor networks revealed that the traffic for similar networks could be modelling by semi-Markov chain [10].

We studying in this paper the multi applications USN and a widely used JPEG will be considered as the compression method. The video amount of data per frame were defined well and have been fit to lognormal, gamma, and Pareto probability distributions or combinations of them [11]. The image amount of data were deter-mined in [5] for monochrome and color images. The probability density functions for both distributions described by Gamma distribution that was confirmed by Kolmogorov-Smirnov criteria.

The mixed telemetry/image USN behaviour in the overload conditions is the investigation goal of this paper. The Hurst coefficient as a function of the nodes number for differences ratio between telemetry and image nodes are obtained by simulation in the ns-2 system. The image nodes share decreases adduce to the decrease the Hurst parameter value at the first step. The Hurst parameter value reduces this then the image nodes share will be too much. It proves that the USN efficiency can reduce in the overload conditions likely the same effect for the PSTN [12] and NGN networks [13].

The results can be enhanced to M2M networks [14].

This article is divided into several parts. The first part presents the results of modelling the USN during increasing the number of nodes - sources of image data. The second part is devoted to the scenario with increasing the number of telemetry nodes, while the sensor field and the number of nodes - sources of image data don't changing. The last part presents conclusions and the future work.

\section{Simulation MODEL AND TOOLS}

The results of previous works proved the self-similar nature of the traffic in the sensor network. But they did not answer to question how the Hurst coefficient depending from different traffic composition.

The sensor field of 30 by 30 meters was selected as base model for investigated USN likely previous experience. The data collection node located in the centre of the field. In according with high interference at the $2.4 \mathrm{GHz}$ broadcast frequency and low power of transmission the guaranteed range of data transmission selected around 15-20 meters. Thereby, the proposed model adequately describes the network with mini-mum number of multi-hop transmission with a single node for data collection. If necessary, the sensor field expansion for data collection by more sink nodes can be placed in parallel.

Two types nodes randomly located in the network. The first type of sensors is nodes that gathering telemetry data, whose task is to transfer small amounts of data with a given period. The transmission frequency is determined for each node type accidentally ranges of 15-60 seconds in steps of 15 seconds. The second type of sensor node is transmitting images. Each 
of these nodes transmits at a random time interval batch of packets that simulate the transfer of photos size of $640 \times 480$ pixels. The amount of data transmitted is randomly selected based on the evaluation of the results get in previous works [5].

The Network simulator 2 system was used for modelling. TCL scripts are generated by scripts in the Python programming language.

\section{III.OVERLOADING BY IMAGE DATA}

In order to analyse the changes of the Hurst coefficient while changing of number of nodes several tests were performed. Maintaining the total number of nodes equal to 55, authors gradually increasing the number of nodes - sources of image data. The number of nodes varied from 5 to 53 in steps of 2 .

The simulation results for selected scenarios with random variables cannot be used to evaluate, so every type of scenario was performed 100 times and the average Hurst coefficient used calculated.

The simulation results are shown in Figure 1, the results for black and white images show as ' $\mathrm{x}$ ' and for colour - as ' $\mathrm{o}$ '.

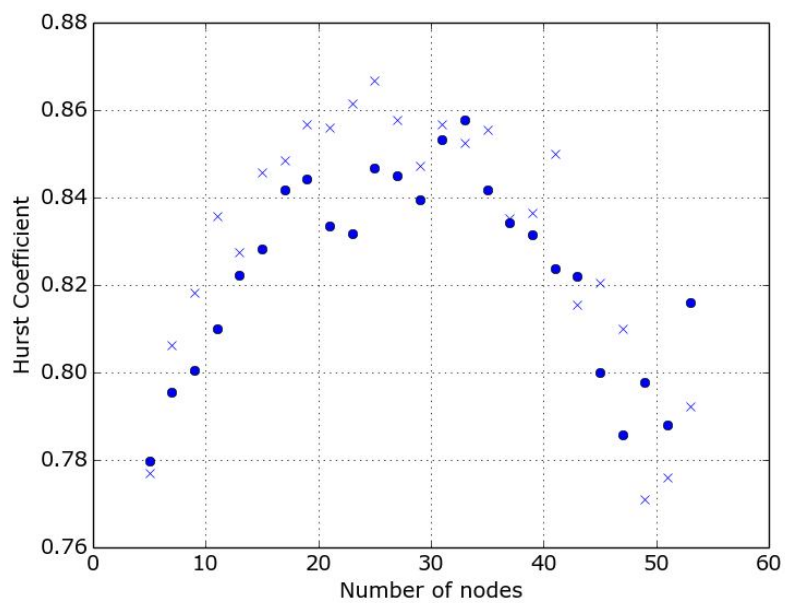

Figure 1. The Hurst coefficient as a function from the number of photo transmitters

As we can see, parabola can be used as a function. Using the mathematical capabilities of the library NumPy the curve parameters were get for the black-and-white and color images. The results are presented in Figure 2 and 3 respectively.

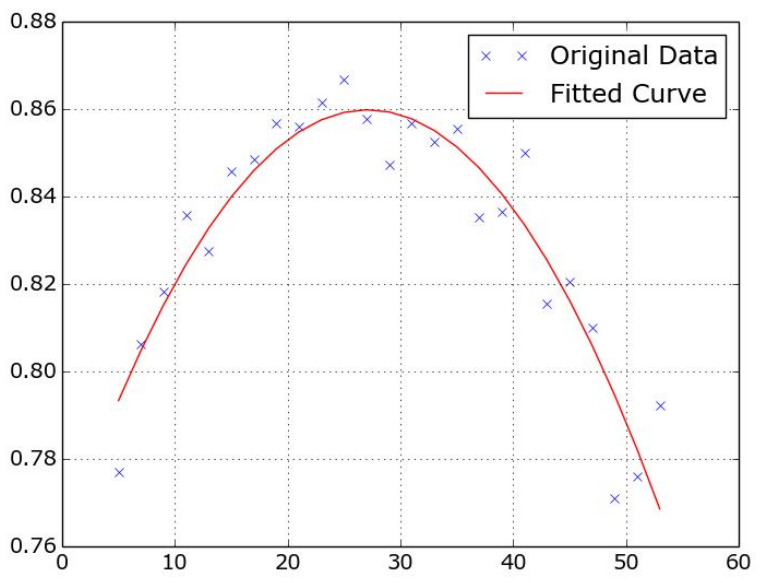

Figure 2. The Hurst coefficient approximation for black and white photo transmitters

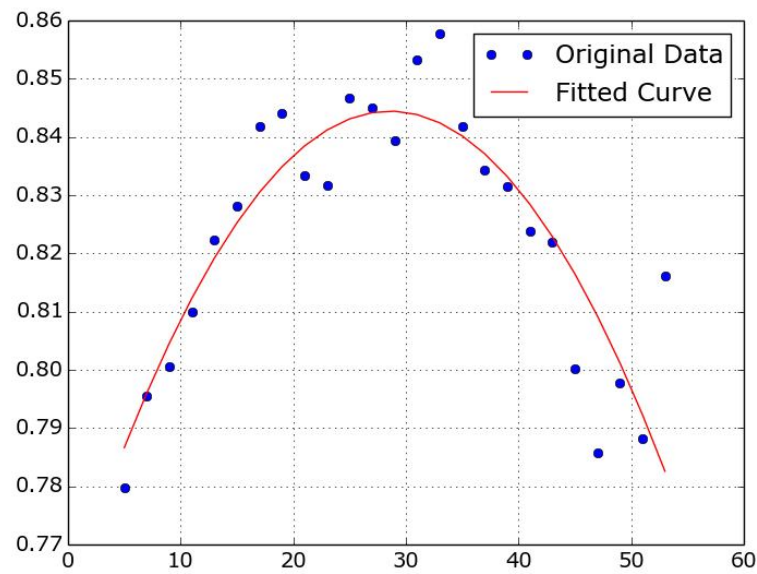

Figure 3. The Hurst coefficient approximation for colour photo transmitters

The overload condition is a state when the network can't handle fully photo transfer. It is easily seen that the Hurst coefficient reaches its maximum in about 30 nodes transmitters. It's clear that the result of changes in the structure of traffic, and there-fore the Hurst coefficient changes caused by network overloading.

We can use the Hurst parameter derivative further. If it is greater than zero means Hurst coefficient is growing and has not reached its maximum. In the case of a nega-tive value of the derivative, we can talk about the problems in the sensor network.

So, the overload control can be made by the Hurst parameter functions derivatives value change. For example we can handle batch of traffic and save calculated Hurst parameters during the time line. Based on Spencer's formulas we can choose the basis of the calculated parameter is it random or matches the distribution. After the derivative changes sign the overload conditions can be defined. 


\section{IV.OVERLOADING BY TELEMETRY DATA}

Second case, examined by authors is an increase the number of nodes collecting telemetry data, while maintaining the size of the sensor field and the number of nodes that transmit the image data don't changing. For this simulation were performed scenarios with increasing total amount of nodes from 20 to 150 for scenarios with $5,10,15$ and 20 nodes - image sources. Each scenario was execute 100 times.

The simulation results for a 5 nodes transmit images are shown in the Figure 4. The results for black and white images are shown as ' $x$ ' and for color as ' $o$ '.

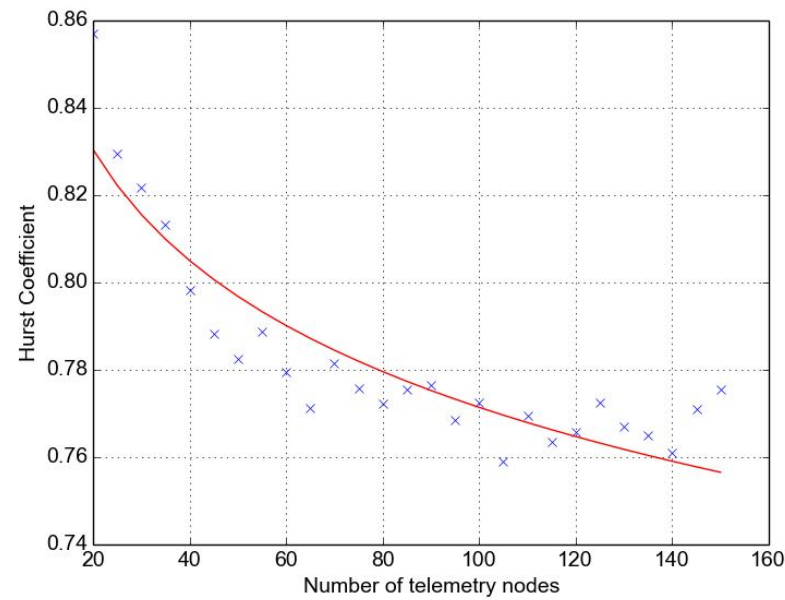

Figure 4. The Hurst coefficient as a function from number of telemetry transmitters

While increasing the number of nodes - sources of telemetry data, load created by nodes - sources of photo ceases to affect the overall structure of the load and the coefficient of Hurst stabilized. The $\log (1 / \mathrm{x})$ function $c a n$ be used. Comparative characteristics for black-and-white images are shown in the Figure 5 and in the Figure 6 for color images. As the number of photo sources and in consequence of the amount of the transmitted load, Hurst coefficient has lower values on all the investigated intervals.

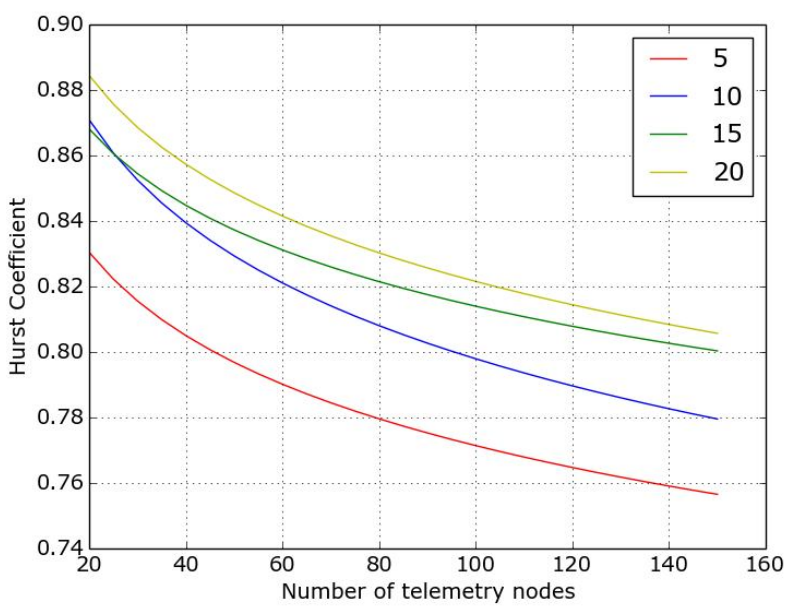

Figure 5. The Hurst coefficient approximation for different number of black and white photo transmitters

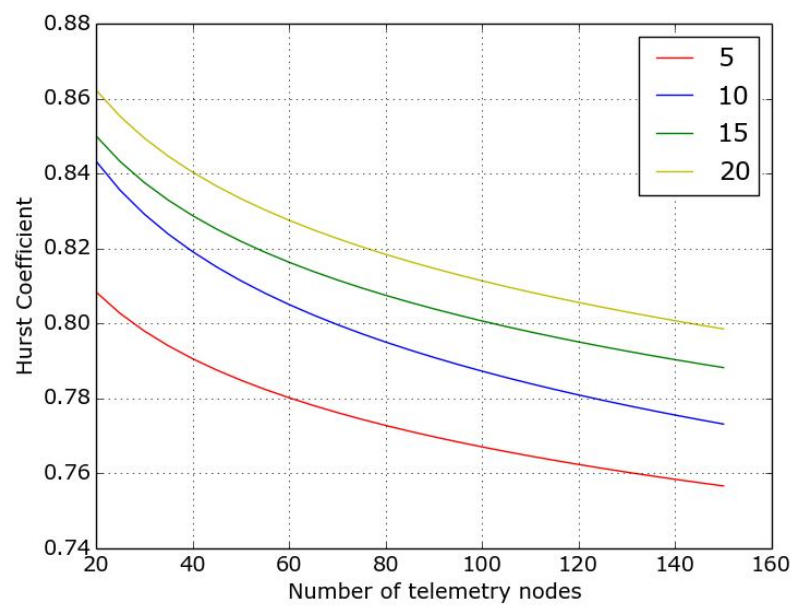

Figure 6. The Hurst coefficient approximation for different number of color photo transmitters

Analyzing the results we can see that the presence of photo traffic lead to bigger value of Hurst coefficient. The increasing the number of telemetry nodes contribution of photo nodes becomes not significant and value of Hurst coefficient decreasing.

\section{Conclusions}

It proves that the USN efficiency can reduce in the overload conditions likely the same effect for the PSTN and NGN networks.

The Hurst coefficient reaches its maximum in about 30 nodes - transmitters. It's clear that the result of changes in the structure of traffic, and therefore the Hurst coefficient changes caused by network overloading. The Hurst coefficient functions derivatives can be used for the USN overload detection.

The photo traffic in mixed USN network has a bigger value of Hurst coefficient. The increasing of the number of telemetry nodes contribution of photo nodes becomes not significant.

The routing in the USN is developed further. The new routing protocol RPL (Routing Protocol for Low energy and lossy networks) [15] became the standard protocol for USN networks. This protocol is designed for IPv6 low power and lossy networks. The directed acyclic graphs are the base for RPL.

Each connection in the RPL network is described by set of metrics such as bit rate, power consumption, encryption support, etc., based on which acyclic graphs are created. In one network possible to create multiple graphs and the node will transmit data depending on the purpose of the data. The graphs for telemetry and image data transmission can be organized by most energy efficient way in the RPL networks.

The future works will include traffic models in the mixed USN network with RPL protocol investigation. 


\section{REFERENCES}

[1] Recommendation Y.2060. Overview of Internet of Things. ITU-T, February 2012, Ge-neva.

[2] Recommendation Y.2062. Framework of object-to-object communication using ubi-quitous networking in NGN. ITU-T, Feb. 2012. Geneva.

[3] A.Koucheryavy, A.Prokopiev. "Ubiquitous Sensor Networks Traffic Models for Telemetry Applications" in The 11th International Conference on Next Generation Wired/Wireless Networking NEW2AN 2011. Aug. 2011 Saint-Petersburg. Springer LNCS 6869.

[4] A.Koucheryavy, A.Vybornova. "Ubiquitous Sensor Networks Traffic Models for Medical and Tracking Applications" in The 12th International Conference on Next Generation Wired/Wireless Networking NEW2AN 2012. Aug. 2012 Saint-Petersburg. Springer LNCS 7469

[5] A.Koucheryavy, A.Muthanna, A.Prokopiev. Ubiquitous Sensor Networks Traffic Models for Image Applications. Internet of Things and its Enablers (INTHITEN). Conference, State University of Telecommunication, St. Petersburg, Russia, June 3-4, 2013. Proceedings.

[6] S.A.Hussain, M.I.Razzak, A.A.Minhas, M.Sher, G.R.Tahiz. "Energy Efficient Image Compression in Wireless Sensor Networks." in International Journal of Recent Trends in Engineering, v.2, N1, Nov. 2009.

[7] H.Wu, A.A.Abouzeid. "Energy Efficient Distributed JPEG 2000 Image compression in Multihop Wireless Networks." in 4th Workshop on Applications and Services in wireless Networks (AWSN), Boston University, USA. Aug. 8-11, 2004.

[8] M.Wu, C.W.Chen. "Collaborative Image Coding and Transmission Over Wireless Sensor Networks." in EURASIP Journal on Advanced in Signal Processing, 2007.

[9] K.-Y.Chow, K.-S.Lui, E.Y.Lam. "Efficient On-Demand Image Transmission in Visual Sensor Networks." in EURASIP Journal on Advanced in Signal Processing, 2007.

[10] Z.Shuai, S.Oh,M.-H.Yang. "Traffic Modeling and Prediction Using Camera Sensor Networks.” in ICDSC 2010, Aug. 31 - Sep. 4, 2010. Atlanta, USA

[11] T.M.Chen. "Network Traffic Modeling." Chapter in The Handbook of Computer Networks. Ed. H.Bidgoli. Wiley, 2007.

[12] M.Schneps. The Information Distribution Systems. The Calculation Methods. Mos-cow, Svyaz, 1979 (in Russian).

[13] Y.Koucheryavy, D.Moltchanov, J.Harju. A Top-down Approach to VoD Traffic Transmission over Diffserv Domain using AF PHB Class. IEEE International Confe-rence on Communications. ICC'03, Anchorage, Alaska, USA, 2003.

[14] S.Andreev, O.Galinina, Y.Koucheryavy. Energy-Efficient Client Relay Scheme for Machine-to-Machine Communication. GLOBECOM 2011, Houston, TX, USA, 2011
[15] Recommendation RFC6550, RPL: IPv6 Routing Protocol for LowPower and Lossy Networks, IETF, March 2012

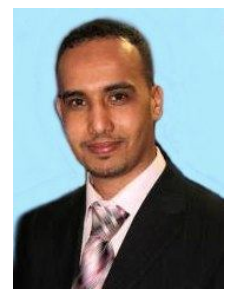

A.Muthanna was born in Lahj, Yemen 01.01.1984. Graduated from St. Petersburg State University of Telecommunications in 2009 bachelor's degree and in 2011 master's degree. $\mathrm{He}$ is $\mathrm{PhD}$ student at"Telecommunication Networks" department.

A.Muthanna's scientific areas of interest are traffic models for ubiquitous sensor networks. In 2012/2013 he take part in Erasmus student program in the University of Ljubljana in Faculty of Electrical engineering.

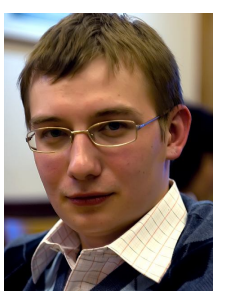

A.Prokopiev was born in small Siberian town Angarsk 11.12.1983. He was studied in St. Petersburg State University of Telecommunication. In 2006 he received Engineer degree. He became PhD in 2012.

After graduating A.Prokopiev works in a small company targeting on the market of wireless sensor networks (WSN), radio frequency identification (RFID) and realtime location system (RTLS). Starting with Engineer position in 2007 , right now he became Head of technical department.

Scope of interests includes Ubiquitous Sensor Networks and RTLS systems. Papers of A. Prokopiev published in science journals in Russia and discussed on international conferences. He is also co-author of book dedicated to wireless self-similar networks.

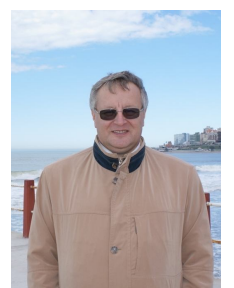

A.Koucheryavy was born in Leningrad 02.02.1952. After graduated from Leningrad University of Telecommunication in 1974 he going to Telecommunication Research Institute named LONIIS, where A.Koucheryavy working up to October 2003 from 1986 up to 2003 as the First Deputy Director ). He became the Ph.D and D.Sc in 1982 and 1994 respectively.

A.Koucheryavy is the St. Petersburg State University of Telecommunication (SUT) professor from 1998. He is SUT department "Telecommunication Networks" chief from 2011. Prof. A.Koucheryavy is the advisor of the Central Science Research Telecommunication Institute and St. Petersburg Branch of "GIPROSVYAZ" Institute simultaneously. He is honorary member of A.S.Popov society.

Prof. A.Koucheryavy was the vice-chairman Study Group 11 ITU-T (Study periods 2005-2008, 2009-2012 ). His scientific areas of interest are the network planning, teletraffic theory, IoT and its enablers. 